\title{
Pharmacological management of post-traumatic seizures in adults: current practice patterns in the UK and the Republic of Ireland
}

\author{
Harry Mee ${ }^{1,2}$ (D) Angelos G. Kolias ${ }^{1,2} \cdot$ Aswin Chari $^{3} \cdot$ Ari Ercole $^{4} \cdot$ Fiona Lecky $^{5} \cdot$ Carole Turner $^{1,2}$. \\ Catrin Tudur-Smith ${ }^{6}$. Jonathan Coles ${ }^{4} \cdot$ Fahim Anwar $^{1}$. Antonio Belli ${ }^{7} \cdot$ Mark Manford $^{1} \cdot$ Timothy Ham $^{1}$. \\ Catherine McMahon $^{8}$. Diederik Bulters ${ }^{9}$. Chris Uff ${ }^{3}$. John S. Duncan ${ }^{10}$. Mark H. Wilson ${ }^{11} \cdot$ Anthony G. Marson $^{12}$. \\ Peter J. Hutchinson ${ }^{1,2}$
}

Received: 24 May 2018 / Accepted: 17 September 2018 / Published online: 1 October 2018

(C) The Author(s) 2018

\begin{abstract}
Background Patient selection for seizure prophylaxis after traumatic brain injury (TBI) and duration of anti-epileptic drug treatment for patients with early post-traumatic seizures (PTS), remain plagued with uncertainty. In early 2017, a collaborative group of neurosurgeons, neurologists, neurointensive care and rehabilitation medicine physicians was formed in the UK with the aim of assessing variability in current practice and gauging the degree of uncertainty to inform the design of future studies. Here we present the results of a survey of clinicians managing patients with TBI in the UK and Ireland.

Materials and methods An online survey was developed and piloted. Following approval by the Academic Committee of the Society of British Neurological Surgeons, it was distributed via appropriate electronic mailing lists.

Results One hundred and seventeen respondents answered the questionnaire, predominantly neurosurgeons (76\%) from 30 (of 32) trauma-receiving hospitals in the UK and Ireland. Fifty-three percent of respondents do not routinely use seizure prophylaxis, but $38 \%$ prescribe prophylaxis for one week. Sixty percent feel there is uncertainty regarding the use of seizure prophylaxis, and $71 \%$ would participate in further research to address this question. Sixty-two percent of respondents use levetiracetam for treatment of seizures during the acute phase, and $42 \%$ continued for a total of 3 months. Overall, $90 \%$ were uncertain about the duration of treatment for seizures, and $78 \%$ would participate in further research to address this question.

Conclusion The survey results demonstrate the variation in practice and uncertainty in both described aspects of management of patients who have suffered a TBI. The majority of respondents would want to participate in future research to help try and address this critical issue, and this shows the importance and relevance of these two clinical questions.
\end{abstract}

This article is part of the Topical Collection on Brain Trauma

Electronic supplementary material The online version of this article (https://doi.org/10.1007/s00701-018-3683-9) contains supplementary material, which is available to authorized users.

Harry Mee

harrymee@nhs.net

1 Department of Clinical Neurosciences, University of Cambridge \& Addenbrooke's Hospital, Cambridge, UK

2 Surgery theme, Cambridge Clinical Trials Unit, Cambridge, UK

3 Department of Neurosurgery, Royal London Hospital, London, UK

4 Neurosciences Critical Care Unit and Division of Anaesthesia, University of Cambridge \& Addenbrooke's Hospital, Cambridge, UK

5 School of Health and Related Research, University of Sheffield, Sheffield, UK
Department of Biostatistics, University of Liverpool, Merseyside, Liverpool, UK

7 Department of Neurosurgery, Queen Elizabeth Hospital, Birmingham, UK

8 Department of Neurosurgery, The Walton Centre, Liverpool, UK

9 Department of Neurosurgery, University Hospital Southampton, Southampton, UK

10 Department of Clinical and Experimental Epilepsy, UCL Institute of Neurology, London, UK

11 Imperial Neurotrauma Centre, Department of Surgery \& Cancer, Imperial College, London, UK

12 Department of Molecular and Clinical Pharmacology, Institute of Translational Medicine, University of Liverpool, Liverpool, UK 
Keywords Post-traumatic seizures $\cdot$ Traumatic brain injury $\cdot$ Anti-epileptic medication $\cdot$ Seizure prophylaxis

\section{Introduction}

Traumatic brain injury (TBI) remains a significant public health problem that can result in physical, cognitive, functional and psychosocial disabilities [7].

Post-traumatic seizures (PTS) are well recognised following TBI. They are typical, albeit somewhat arbitrarily, classified as immediate (at time of impact), early (within 7 days post-TBI) or late (after 7 days) [5]. Seizures during acute hospitalisation can lead to significant derangement of brain physiology, contributing to secondary injury through energetic crisis and/or intracranial hypertension or even directly leading to brain herniation and death. Additionally, PTS during acute hospitalisation has been shown to be an independent risk factor for PTS within 12 and 24 months following TBI [9]. Late PTS can have a negative impact on quality of life, return to work, return to driving and can even result in death. The rationale for seizure prophylaxis with an anti-epileptic drug (AED) during acute hospitalisation is that the incidence of early PTS in patients following severe TBI is as high as $14 \%$ [12] and prevention of seizures can limit derangements in brain physiology, lower the risk of herniation and death and potentially prevent the development of late PTS. However, AEDs have variable positive, negative or neutral effects in both cognitive and behavioural domains [8]. They are also associated with some other side effects including bone density loss, hepatotoxicity and StevensJohnson Syndrome [4]. It is therefore essential to ensure that AEDs are prescribed appropriately and for the optimal duration following TBI.
Many studies of seizure prophylaxis pre-date the availability of EEG monitoring in the ITU, and in the light of evidence of the frequency of subclinical seizures in TBI, this question would benefit from reevaluation [3]. Patients who develop PTS in the acute phase after a TBI are typically started on an AED to prevent further seizures. However, there is no highquality evidence regarding the optimal duration of treatment for this group of patients.

There is a pressing need for high-quality evidence, and a baseline understanding of clinical practice is an essential pre-requisite for the design of an appropriate clinical trial. In 2017, a collaborative group of neurosurgeons, neurointensive care physicians and rehabilitation medicine physicians was formed with the aim of examining current practice patterns, gauging the degree of uncertainty and thus designing relevant future studies on the use of AEDs following TBI. It was agreed that a questionnaire survey would be a pragmatic way of achieving the first two objectives.

\section{Materials and methods}

In line with the above objectives, we developed and piloted a questionnaire survey. Subsequently, the questionnaire survey was approved by the academic committee of the Society of British Neurological Surgeons (SBNS). A convenience sample of clinicians with interest in the management of patients with TBI and/or seizures was asked to complete the survey. A
Fig. 1 Responses to question: 'Which anti-epileptic drug do you use as first choice for seizure prevention (i.e. the patient has not had a seizure) in moderate to severe traumatic brain injury during the acute phase?'

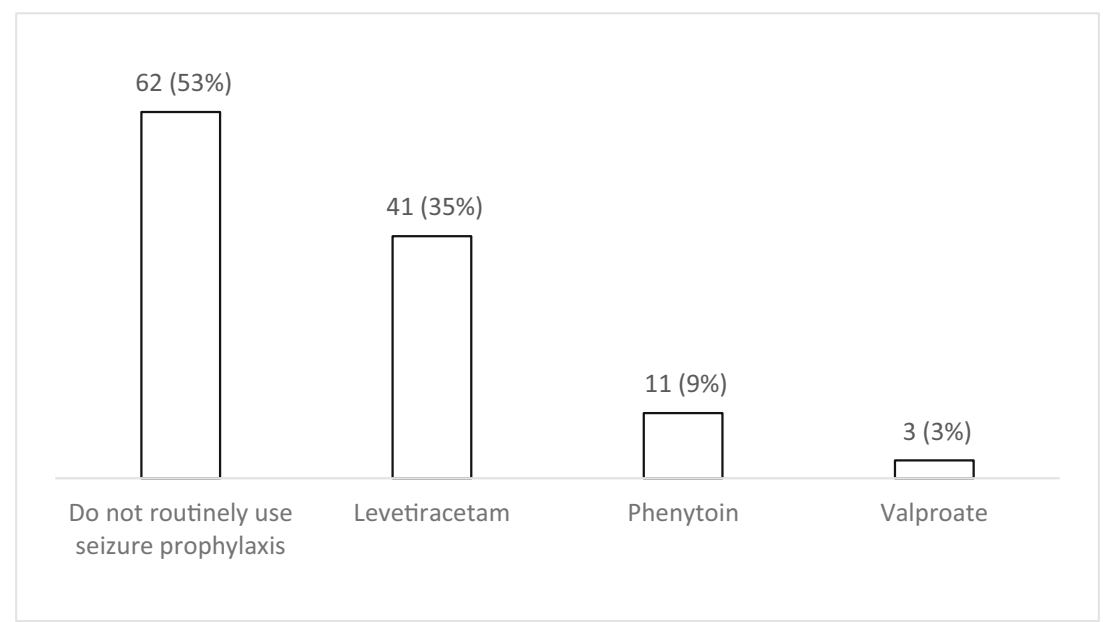


Fig. 2 Responses to question: 'Which factors influence your decision to start seizure prophylaxis?' (can select more than one answer)

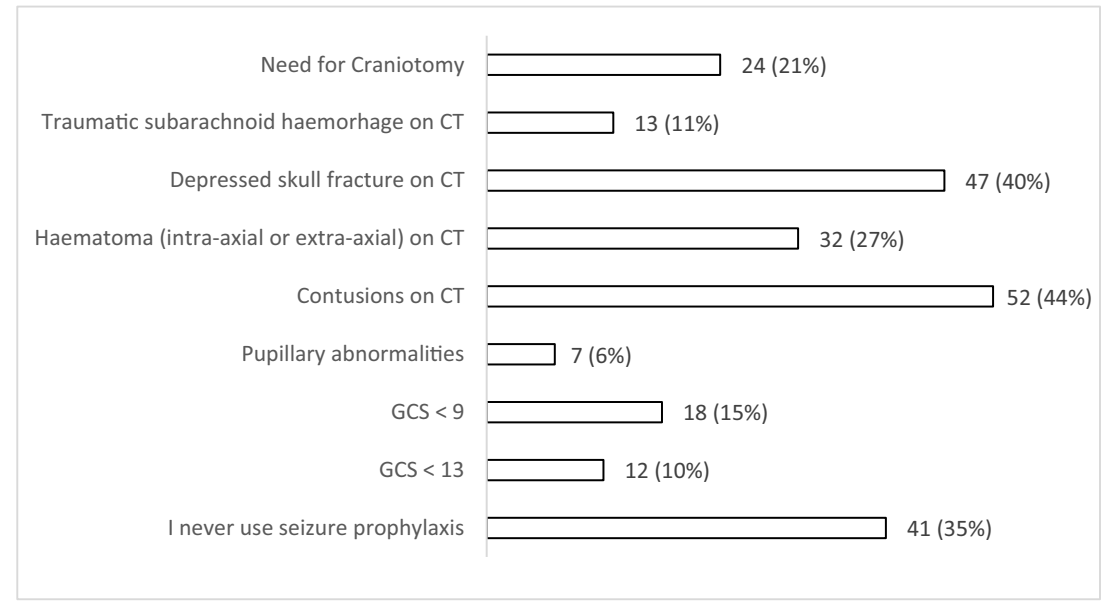

secure online survey tool was used to disseminate the questionnaires via the electronic mailing lists of the SBNS, British Neurosurgical Trainees Association (BNTA) and included in the Association of British Neurologists newsletter. The survey was also promoted by the Twitter accounts of the SBNS (@The_SBNS),BNTA (@e1v1m1), British Neurotrauma Group (@bntg_uk), British Neurosurgical Trainee Research Collaborative (@BNTRC) and Association of British Neurologists (@theABN_Info).

Our target audience were clinicians who were involved with the acute and long-term management of TBI patients, who were linked with adult traumareceiving neurosurgical units. We disseminated the survey to neurousurgeons, intensive care medicine/anaesthesia, neurology, emergency medicine and rehabilitation medicine. Due to the wide dissemination of the questionnaire through social media platforms, calculation of the response rate is not possible; $95 \%$ confidence intervals have been used and documented as (\%-\%) after the figures.

\section{Results}

The online questionnaire was completed by 117 clinicians from a range of specialties, but predominately neurosurgeonneurosurgery $(n=89,76 \%)$, intensive care medicine/ anaesthesia $(n=24,21 \%)$, neurology $(n=2,2 \%)$, emergency medicine and rehabilitation medicine ( $n=1$ each). The majority of the respondents were consultants $(n=78,67 \%)$, while the remaining were trainees or fellows $(n=39,33 \%)$. There were respondents from 30 of the adult trauma-receiving neurosurgical units, 29 in the UK and 1 from Ireland. There was at least 1 response from a Consultant from 21/30 (66\%) of the adult receiving neurosurgical units. The questionnaire disseminated can be found in the online supplementary material.

\section{Seizure prophylaxis}

Fifty-three percent $(n=62 ; 44-62 \%)$ of respondents do not use seizure prophylaxis routinely compared to $47 \%(n=55$;
Fig. 3 Responses to question: 'If you start seizure prophylaxis during the acute phase, how long do you continue (assuming that no seizures occur)?'

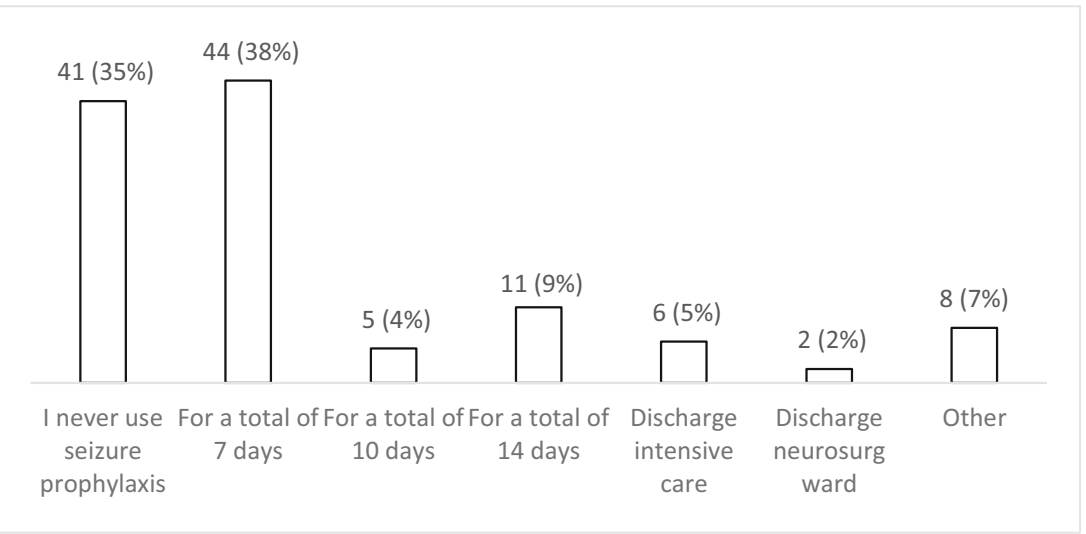


Fig. 4 Responses to question: 'Do you think that there is uncertainty/equipoise about the use (or not) of seizure prophylaxis in moderate to severe traumatic brain injury during the acute phase?'

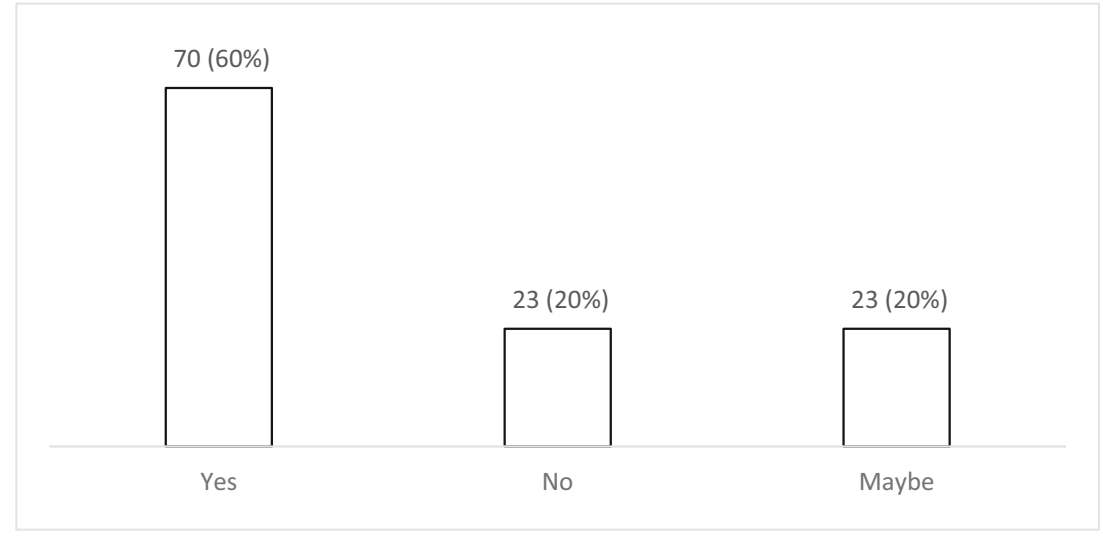

31-50\%) who do so for patients with a moderate or severe TBI during the acute phase (Fig. 1). Of those who use prophylaxis, $75 \%(n=41 / 55 ; 61-85 \%)$ chose levetiracetam over phenytoin $(n=11 / 55 ; 20 \%(11-33 \%))$ or valproate $(n=3 / 55$; $5 \%(1-16 \%)$. When asked about factors influencing their decision to start prophylaxis (Fig. 2), 65\% of the respondents $(n=76 ; 55-73 \%)$ selected at least one factor. The top five factors influencing the decision to start seizure prophylaxis are the presence of contusions on CT $(n=52)$, depressed skull fracture $(n=47)$, intra-axial or extra-axial haematoma on CT $(n=32)$, need for craniotomy $(n=24)$ and a GCS $<9(n=18)$. When asked about the length of prophylaxis, the majority $(n=44 ; 58 \%$ (29-47\%)) prescribe prophylaxis for 7 days (Fig. 3). Finally, the majority $(n=70 ; 60 \%(50-69 \%))$ felt that there is uncertainty/equipoise surrounding the use of seizure prophylaxis (Fig. 4) with $71 \%(n=83 ; 62-79 \%)$ stating that they would participate in a randomised trial to address seizure prophylaxis in moderate to severe TBI during the acute phase (Fig. 5).

\section{Treatment of early PTS}

The majority of respondents $(n=72 ; 62 \%(52-70 \%))$ use levetiracetam for patients with PTS during the acute phase (Fig. 6). Nearly one third $(n=35 ; 30 \%(22-39 \%))$ use phenytoin with valproate favoured by less than $10 \%(n=8 ; 3-$ $13 \%)$. There was variation in the duration of treatment with AEDs (Fig. 7), with $42 \%(n=49 ; 33-51 \%)$ continuing treatment for 3 months if no further seizures occur, $24 \%(n=28$; $17-33 \%)$ for 6 months, $10 \%(n=12 ; 5-18 \%)$ for 12 months and $12 \%(n=14 ; 7-20 \%)$ tapering after discharge from the hospital (Fig. 7). Ninety percent $(n=105 ; 82-94 \%)$ stated that there is uncertainty regarding the optimal duration of treatment with AEDs for PTS occurring during acute hospitalisation (Fig. 8), with 78\% ( $n=91 ; 69-85 \%)$ stating that they would participate in a randomised trial to address duration of treatment (Fig. 9).

When respondents were asked to select the top priority for future research in the field of PTS, 57\% $(n=67 ; 48-66 \%)$
Fig. 5 Responses to question: 'Would you participate in a randomised trial to address seizure prophylaxis in moderate to severe traumatic brain injury during the acute phase?'

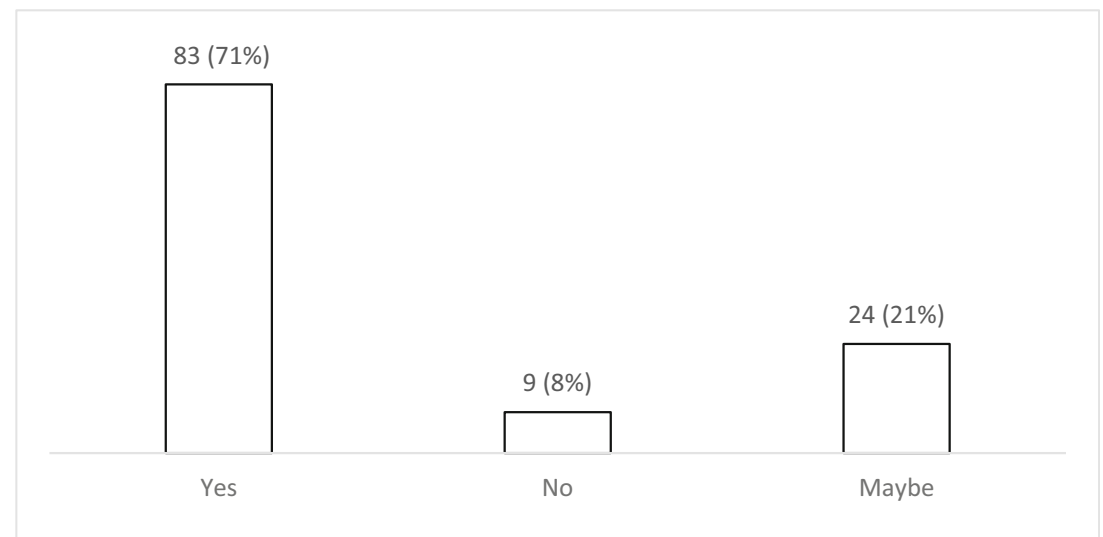


Fig. 6 Responses to question: 'Which anti-epileptic drug do you use for a patient with traumatic brain injury who has had seizure(s) during the acute phase?'

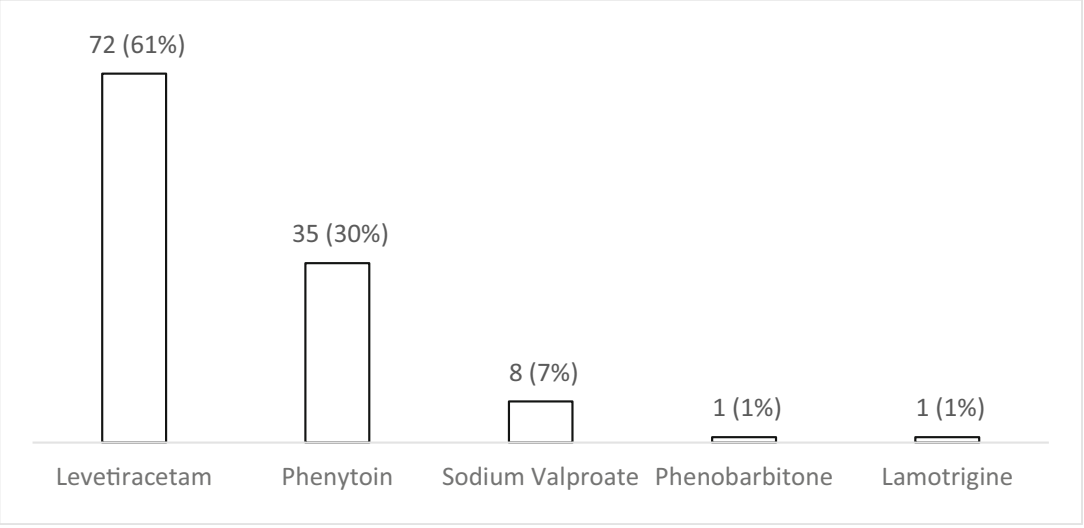

answered seizure prophylaxis, nearly one third $(n=37 ; 32 \%$ (24-41\%)) duration of treatment for PTS during the acute phase, and $10 \%$ favoured research on the type of AEDs that should be used (Fig. 10).

\section{Discussion}

The survey findings confirm that there is significant variation in the practice across the UK and Ireland with regard to the use of seizure prophylaxis and the duration of treatment with AEDs after early PTS. A Cochrane review [14], concluded that there is 'low-quality evidence that early treatment with an AED compared with placebo or standard care reduced the risk of early post-traumatic seizures' and that 'there was no evidence to support a reduction in the risk of late seizures or mortality'. Despite that, nearly half of the respondents routinely use prophylactic AEDs (47\%). The 2016 'Brain Trauma Foundation' guidelines [1] stated that 'phenytoin is recommended to decrease the incidence of early PTS, when the overall benefit is felt to outweigh the complications associated with such treatment', but concluded that 'there was insufficient evidence to support a Level I recommendation for the topic of post-traumatic seizures' and are calling for further trials.

The survey showed that the two most commonly used AEDs, for prophylaxis or treatment, are levetiracetam and phenytoin with the former having surpassed the latter in popularity. This reflects the findings of a recent survey of US clinicians [10], which showed that $74 \%$ of the respondents prefer levetiracetam for seizure prophylaxis, with only $10 \%$ favouring phenytoin. A similar trend has also recently been demonstrated in Europe [6].

Temkin et al. [12] demonstrated that phenytoin given for 1-year versus placebo decreased early PTS (within 7 days) from $14.2 \%$ down to $3.6 \%$, but seizure rate did not vary after 7 days. Therefore, the available evidence, so far, suggests prophylaxis treatment is beneficial for reduction of early PTS only. Our study shows a variable prophylaxis rate, with $52 \%$ not using prophylaxis routinely and $60 \%$ being uncertain about the use of prophylaxis. A prospective, randomised, single-blinded study by Szaflarski et al. [11] showed no difference between the seizure rates of phenytoin or levetiracetam. However, this was a small study, and it is noted that further exploration is required. Due to its superior side effect profile and the fact, there is no need for plasma
Fig. 7 Responses to question: 'If you initiate treatment with antiepileptics for seizures in the acute phase after traumatic brain injury, how long do you continue for?'

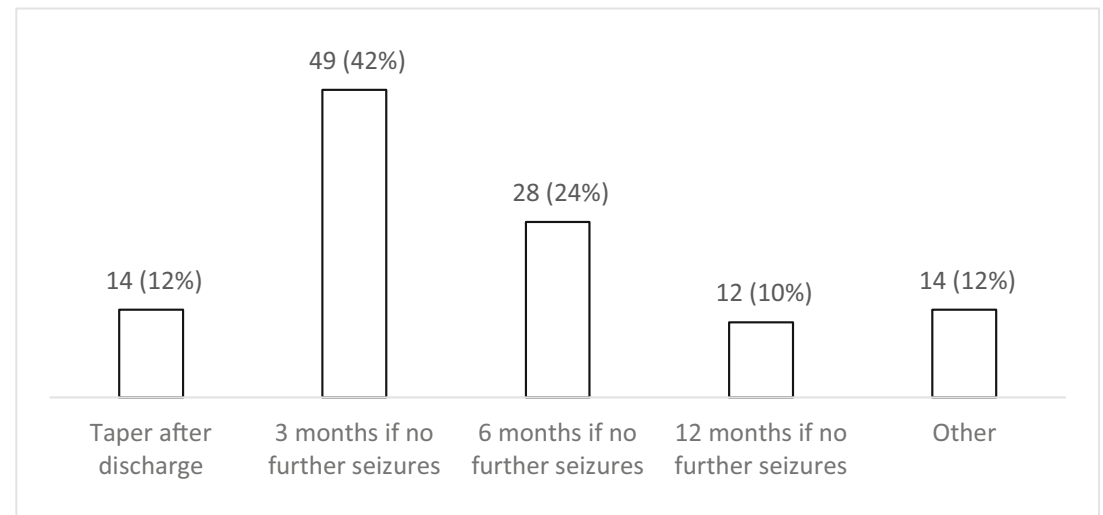


Fig. 8 Responses to question: 'Do you think that there is uncertainty about the duration of treatment with anti-epileptic drugs for seizures occurring in the acute phase after traumatic brain injury?'

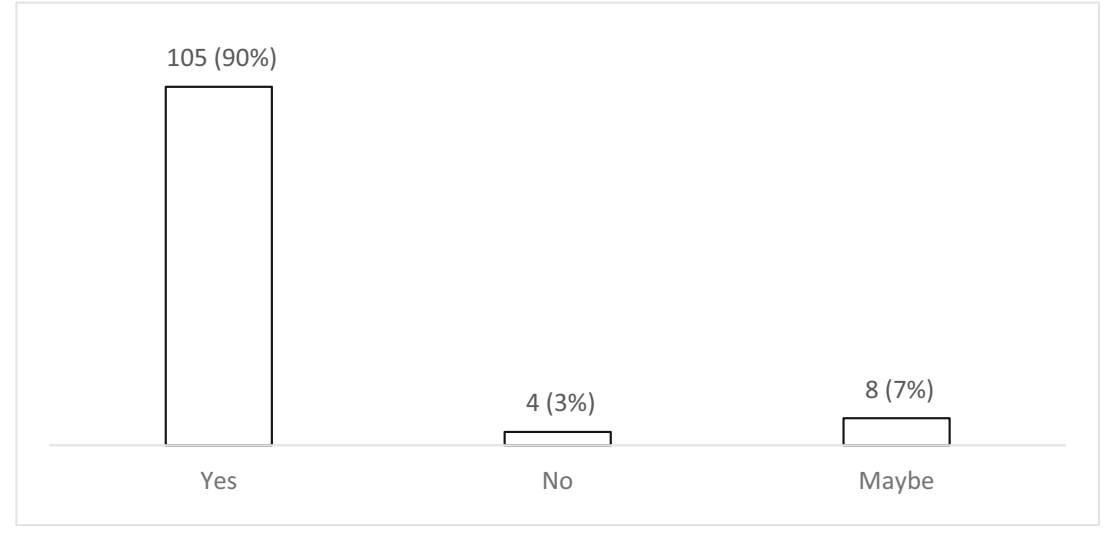

monitoring levetiracetam has become the AED of choice, with $58 \%$ of respondents choosing to use this drug.

PTS during and after acute hospitalisation are often harmful. Recurrent PTS post-TBI can negatively impact on quality of life, return to work/driving and can even lead to death. PTS during acute hospitalisation has been shown to be an independent risk factor for PTS within 12 and 24 months following TBI [9]. AEDs are the mainstay of treatment for patients with PTS but are associated with side effects that, if serious, can negatively impact on quality of life, cognition and general health [4, 8]. Patients with acute PTS are typically started on an AED to prevent seizure recurrence. The optimal duration of treatment remains unclear [13] but as TBI carries an increased risk of epilepsy as a consequence of recurrent seizures [2], further trials are necessary to try and answer these important questions.

Although we acknowledge there are limitations in questionnaire surveys and appreciate that the response rate of online surveys is not possible to know due to the multiple channels of dissemination, we feel that having over 100 responses from the majority of adult trauma-receiving neurosurgical units in the UK and Ireland provides a reasonable overview of the current practice patterns. A further limitation is the fact that there were only Consultant responses from two thirds of the units; however, trainees and speciality doctors in these units play an active role in the management of TBI patients and PTS and therefore the value of having their views cannot be ignored and commonly will reflect the views of the consultants.

The survey results demonstrate that there is significant uncertainty as to the duration of treatment of acute PTS, and also, uncertainty surrounding whether prophylaxis for PTS should be given. The results of the survey are not surprising as they underline the known uncertainity of current practices across the UK and Ireland and confirms the need for future research around this topic.

The uncertainties are most likely due to the lack of highquality data investigating the duration of treatment and prophylaxis of PTS. The fact that the majority of the respondents
Fig. 9 Responses to question: 'Would you participate in a randomised trial to address duration of treatment with antiepileptic drugs for seizures occurring in the acute phase after traumatic brain injury?'

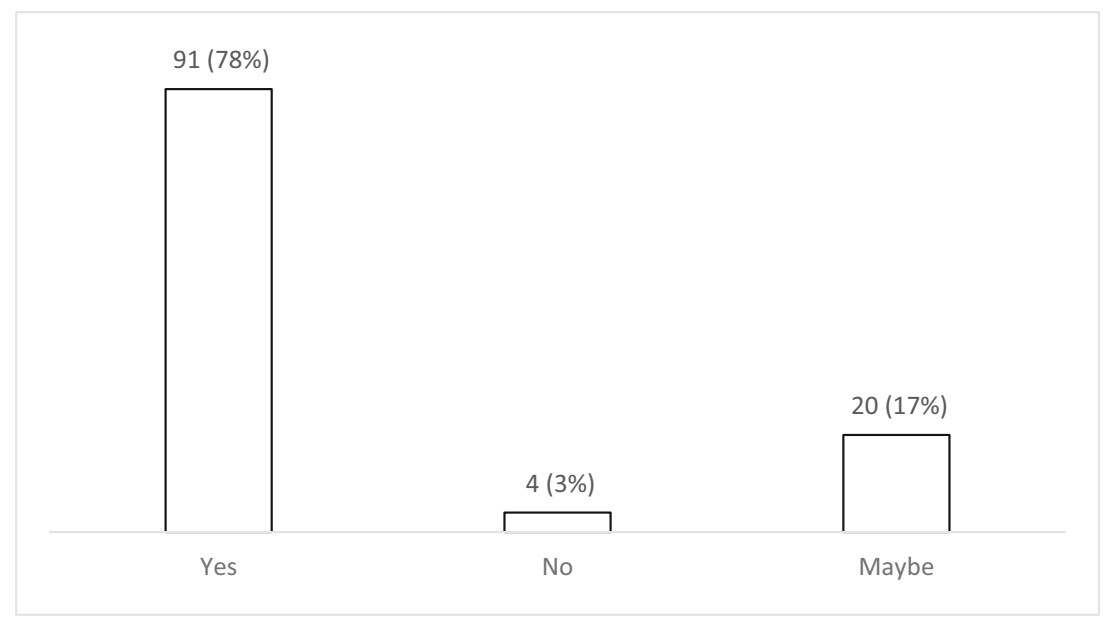


Fig. 10 Responses to question: 'There are a few uncertainties in the management of post-traumatic seizures that could be addressed by future studies. Which one is the most important priority in your opinion?'

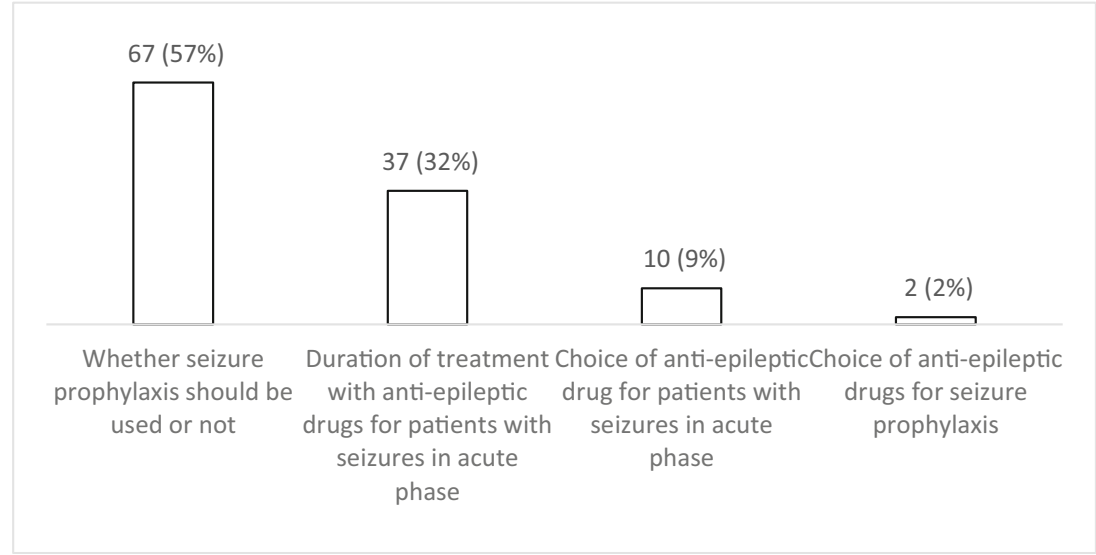

are willing to collaborate on future studies highlights the importance of this subject to the community of clinicians caring for TBI patients in the UK.

\section{Conclusions}

The current paper demonstrates the variation in practice and uncertainty in both described aspects of the management of patients with TBI. The majority of respondents would want to participate in future research to help try and address these issues, and this shows the importance and relevance of these two clinical questions. Ultimately, class I evidence is necessary to provide clinicians with a better evidence base and achieve further improvements in the outcome of patients with TBI and PTS.

Acknowledgements Angelos Kolias is supported by a Clinical Lectureship, School of Clinical Medicine, University of Cambridge. Peter Hutchinson is supported by a Research Professorship from the National Institute for Health Research (NIHR), the NIHR Cambridge Biomedical Research Centre, a European Union Seventh Framework Program grant (CENTER-TBI; grant no. 602150) and the Royal College of Surgeons of England.

We thank the academic committee of the SBNS for reviewing and approving the questionnaire.

\section{Compliance with ethical standards}

The authors alone are responsible for the content and writing of the paper. This article does not contain any studies with human participants performed by any of the authors.

Conflict of interest All authors certify that they have no affiliations. with or involvement in any organisation or entity with any financial interest (such as honoraria; educational grants; participation in speakers' bureaus; membership, employment, consultancies, stock ownership, or other equity interest; and expert testimony or patent-licencing arrangements), or non-financial interest (such as personal or professional relationships, affiliations, knowledge or beliefs) in the subject matter or materials discussed in this manuscript.
Open Access This article is distributed under the terms of the Creative Commons Attribution 4.0 International License (http:// creativecommons.org/licenses/by/4.0/), which permits unrestricted use, distribution, and reproduction in any medium, provided you give appropriate credit to the original author(s) and the source, provide a link to the Creative Commons license, and indicate if changes were made.

\section{References}

1. Carney N, Totten A, OReilly C et al (2016) Guidelines for the management of severe traumatic brain injury. 1:1-244

2. Christensen J (2012) Traumatic brain injury: risks of epilepsy and implications for medicolegal assessment. Epilepsia 53(Suppl. 2): 43-47. https://doi.org/10.1111/j.1528-1167.2012.03612.x

3. Claassen J, Mayer SA, Kowalski RG, Emerson RG, Hirsch LJ (2004) Detection of electrographic seizures with continuous EEG monitoring in critically ill patients. Neurology 62:1743-1748

4. EK SL (2009) Minimizing AED adverse effects: improving quality of life in the interictal state in epilepsy care. Curr Neuropharmacol: 106-114

5. Frey LC (2003) Epidemiology of posttraumatic epilepsy: a critical review. Epilepsia 44:11-17

6. Huijben JA, Volovici V, Cnossen MC, et al. (2018) Variation in general supportive and preventive intensive care management of traumatic brain injury: a survey in 66 neurotrauma centers participating in the Collaborative European NeuroTrauma Effectiveness Research in Traumatic Brain Injury (CENTER-TBI) study. 1-9. https://doi.org/10.1186/s13054-018-2000-6

7. Maas PAIR, Menon PDK, Adelson PD, The Lancet Neurology Commission et al (2017) Traumatic brain injury: integrated approaches to improve prevention, clinical care, and research. Lancet Neurol 16(12):987-1048. https://doi.org/10.1016/S14744422(17)30371-X

8. Nadkarni S, Devinsky O (2005) Psychotropic effects of antiepileptic drugs. Clin Sci 5(5):176-181

9. Ritter AC, Wagner AK, Szaflarski JP et al (2016) Prognostic models for predicting posttraumatic seizures during acute hospitalization, and at 1 and 2 years following traumatic brain injury. Epilepsia 57(9):1503-1514. https://doi.org/10.1111/epi.13470

10. Szaflarski JP (2015) Is there equipoise between phenytoin and levetiracetam for seizure prevention in traumatic brain injury? Epilepsy Curr 15:94-97

11. Szaflarski JP, Sangha KS, Lindsell CJ, Shutter LA (2009) Prospective, randomized, single-blinded comparative trial of intravenous levetiracetam versus phenytoin for seizure prophylaxis. 
Neurocrit Care 12(2):165-172. https://doi.org/10.1007/s12028009-9304-y

12. Temkin NR, Dikmen SS, Wilensky AJ, Keihm J, Chabal S, Winn R (1990) A randomized, double-blind study of phenytoin for the prevention of post-traumatic seizures. N Engl J Med 323:497-502

13. Thompson K, Pohlmann-Eden B, Campbell LA, Abel H. (1996) Pharmacological treatments for preventing epilepsy following traumatic head injury. (Thompson K, ed.). Chichester: John Wiley \& Sons, Ltd; 1-3. doi:https://doi.org/10.1002/14651858.CD009900. pub2

14. Thompson K, Pohlmann-Eden B, Campbell LA, Abel H (2015) Pharmacological treatments for preventing epilepsy following traumatic head injury. Thompson K, ed. Cochrane Database Syst Rev 26(8):1-83. https://doi.org/10.1002/14651858.cd009900.pub2

\section{Comments}

We have read with much interest the recent paper by Mee et al. [1].

The authors presented the results of a survey of clinicians managing patients with traumatic brain injury (TBI) in the UK and Ireland. The survey results demonstrate the variation in practice and uncertainty both in patient selection for seizure prophylaxis and in the duration of antiepileptic drug treatment in early post-traumatic seizures (PTS). Moreover, it clearly shows the necessity of future research on the topic.
First, we would like to congratulate the authors for the well-written presentation of this survey results as we believe they are very informative and, providing a practical overview of the current clinical practice, highlight the uncertainties in the management of PTS. Moreover, we would like to stress further the real and strong necessity of additional effort in this field, most of all in the light of the recent gain in understanding of underlying mechanisms subtending seizures. In respect of great resonance in the field is the update of Classification of the Epilepsies recently performed by the International League Against Epilepsy (ILAE) [2]. The new classification incorporates etiology along each stage, emphasizing the need to consider etiology at each step of diagnosis, as it often carries significant treatment implications. It is likely that that this new classification will assist in improving epilepsy care and research in many clinical contests, surely including the TBI

Domenico d'Avella

Florinda Ferreri

Padova, Italy

Bibliography

1. Mee et al...ACTA Neurochirurgica

2. Scheffer IE, Berkovic S, Capovilla G, Connolly MB, French J, Guilhoto L, Hirsch E, Jain S, Mathern GW, Moshé SL, Nordli DR, Perucca E, Tomson T, Wiebe S, Zhang YH, Zuberi SM. ILAE classification of the epilepsies: Position paper of the ILAE Commission for Classification and Terminology. Epilepsia. 2017 Apr;58(4):512-521. doi: 10.1111/epi.13709. Epub 2017 Mar 8 